\title{
Association of serum concentrations of perfluoroalkyl compounds with poor growth and failure to weight gain in 2-year-old children
}

Young Ah Lee ${ }^{1 *}$, Jin Hee Kim ${ }^{2,3}$, Hwa Young Kim', Haewoon Jung ${ }^{1}$, Jieun Lee ${ }^{1}$, Juyoung Yoon ${ }^{1}$, Sanghyuk Bae ${ }^{2,4}$, Yun-Chul Hong ${ }^{2,3,4}$, Choong Ho Shin', Sei Won Yang ${ }^{1}$

From 8th APPES Biennial Scientific Meeting

Darwin, Australia. 29 October - 1 November 2014

\section{Backgrounds}

Potential health concerns of perfluoroalkyl compounds (PFCs) have been raised.

\section{Objectives}

We investigated the relationship between exposure to PFCs and growth parameters in Korean 2-year-old children.

\section{Methods}

Three hundred sixty children (189 boys, 1.9 to 2.2 years) born as appropriate gestational age infants were enrolled. Height and weight at visit, birth weight, midparental height $(\mathrm{MPH})$ and bone age (BA) were evaluated.

\section{Results}

Among fifteen PFCs analyzed, perfluorohexane sulfonic acid (PFHxS), and perfluorooctane sulfonic acid (PFOS), perfluorooctanoic acid (PFOA), perfluorononanoic acid (PFNA), and perfluorodecanoic acid (PFDA) were detected in $>90 \%$ of the serum samples. The number of chemicals above median concentrations among these 5 PFCs were graded on a scale of 0 to 5 , and classified into exposure (0) ( $\mathrm{n}=97)$, exposure $(1-2)(\mathrm{n}=88)$, and exposure $(\geq 3)$ groups. After adjusting for sex, birth weight, MPH, and BA, log-transformed PFHxS, PFOS, PFOA, and PFDA were associated with a 1.60, 1.35, $1.57,1.29 \mathrm{~cm}$ decrease in height (all $\mathrm{P}<0.005$ ). Logtransformed PFOS, PFOA, PFNA, and PFDA were negatively related to weight gain (all $\mathrm{P}<0.05$ ). Change in weight Z-scores decreased progressively from exposure
(0), to exposure (1-2), and to exposure ( $\geq 3)($ mean +0.43 vs. +0.29 vs. $+0.10, \mathrm{P}=0.012$ ).

\section{Conclusions}

Increased concentrations of PFOS, PFOA, PFNA, and PFDA were associated with short stature and failure to weight gain in 2-year-old children. The more PFCs detected above median concentrations, the shorter and the poorer weight gain. Further prospective studies are needed to clarify causal relationship.

\section{Authors' details \\ 'Department of Pediatrics, Seoul National University Children's Hospital, Seoul, Korea. 'Environmental Health Center, Seoul National University College of Medicine, Seoul, Korea. ${ }^{3}$ Department of Environmental Health, Graduate School of Public Health, Seoul Nati, Korea. ${ }^{4}$ Institute of Environmental Medicine, Seoul National University Medical Research, Seoul, Korea.}

Published: 28 April 2015

\section{doi:10.1186/1687-9856-2015-S1-P31}

Cite this article as: Ah Lee et al:: Association of serum concentrations of perfluoroalkyl compounds with poor growth and failure to weight gain in 2-year-old children. International Journal of Pediatric Endocrinology 2015 2015(Suppl 1):P31. 